\title{
A new integral transform and associated distributions
}

\begin{abstract}
In this paper, we generalize the concepts of a new integral transform, namely the Sumudu transform, to distributions and study some of their properties. Further, we also apply this transform to solve one-dimensional wave equation having a singularity at the initial conditions.
\end{abstract}

Keyword: Integral transforms; Sumudu transform; PDE(s) with variable coefficients; Double convolution; Distributions 